\title{
Adenosine Deaminase - a Novel Diagnostic and Prognostic Biomarker for Oral Squamous Cell Carcinoma
}

\author{
Deepak Chandrakant Kelgandre ${ }^{1 *}$, Jigna Pathak ${ }^{1}$, Shilpa Patel ${ }^{2}$, Pramod Ingale ${ }^{3}$, \\ Niharika Swain ${ }^{1}$
}

\begin{abstract}
Background: The number of patients with oral cancer in India is increasing gradually (especially in younger people). Although the diagnostic modalities and therapeutic management of oral cancer are improving, the treatment outcome and prognosis of oral cancer remain poor. The absence of definite early warning symptoms for most head and neck cancers suggests that sensitive and specific biomarkers are likely to be important in screening for high-risk patients. Aims: To analyze serum adenosine deaminase (ADA) levels in oral squamous cell carcinoma (OSCC) cases who reported to our institute. Materials and Methods: A prospective study was performed on 100 histopathologically proven cases of OSCC (study group) and 100 normal healthy individuals (control group). Independent sample and one sample t-tests and one way ANOVA followed by Tuckey's POST HOC test were conducted for analysis. Results: Statistically significant increase in serum ADA levels was observed in OSCC cases compared to the control group. Also serum ADA level increased significantly with the histopathological grade. Conclusions: Serum ADA levels in OSCC may be a useful diagnostic and prognostic biomarkers in clinical practice and our findings suggest that a large-scale study is warranted to confirm clinical utility as a prognostic and diagnostic biomarker.
\end{abstract}

Keywords: Oral SCC - adenosine deaminase - biomarker - prognosis

Asian Pac J Cancer Prev, 17 (4), 1865-1868

\section{Introduction}

Failure or delay in the early diagnosis of oral squamous cell carcinoma (OSCC) is one of the primary causes of high mortality and morbidity in cancer patients (Ferlay et al., 2004). Further insights into the mechanisms leading to malignancy are prerequisite for identifying new biomarkers for OSCC from the serum. The idea of screening and following patients with malignancy by serum analysis is appealing from several points of view including its ease, economical advantage, noninvasiveness and possibility of repeated sampling.

Serum ADA level is shown to be increased in cancers of many tissues like colon, bladder, breast, esophagus and liver. Therefore aim of the study was to analyze Serum ADA level in OSCC and objectives of the study were to compare serum ADA level of OSCC patients with normal healthy individuals, to correlate serum ADA level of OSCC patients with histopathological grades of OSCC and to determine the diagnostic and prognostic implications of serum ADA level in OSCC patients.

\section{Materials and Methods}

In the present prospective study $(n=90)$ OSCC patients who reported to our institute were selected as a study group. Detailed case history of all the patients was taken. Exclusion criteria includes cases having history of prior treatment for OSCC or other malignancies, Cases suffering from granulomatous diseases like tuberculosis and cases suffering from any other malignancy. Incisional biopsy was performed in clinically suspected cases of Oral carcinomas under local anesthesia by using $2 \%$ Lignocaine.

Tissue samples were processed by routine method and paraffin embedded blocks was made. Paraffin embedded specimen was cut into sections of 5 micrometer thickness by using the soft tissue microtome and sections were stained by using hematoxylin and eosin stain. According to descriptive criteria for OSCC grading provided by Royal College of Pathologists and WHO 2005, patients were categorized into 3 grades i.e. well differentiated OSCC, moderately differentiated OSCC and poorly differentiated 


\section{OSCC.}

The grading is based on the following Criteria: Degree of Keratinization, Cellular differentiation, Nuclear Changes, lymphoplasmablastic infiltration and the Invasive front. Based on the above mentioned criteria, study comprised $(n=30)$ well differentiated, $(n=30)$ moderately differentiated and $(n=30)$ poorly differentiated OSCC cases. 90 age matched healthy individuals who had no history of any malignancy and infectious conditions in past six months were selected as a control group. $5 \mathrm{ml}$ venous blood was collected from both the groups under the aseptic conditions after taking patients consent. Serum was separated by using centrifugation machine. Serum analysis for ADA was done in both the groups.

\section{Adenosine deaminase assessment}

Estimation of serum ADA level was done by using calorimetric method of Galanti and Guisti (Guesti G, 1974). Adenosine Deaminase hydrolyses adenosine to ammonia and inosine. The ammonia further formed reacts with a phenol and hypochlorite in an alkaline medium to form a blue indophenol complex with sodium nitroprusside acting as a catalyst. Intensity of blue colored indophenol complex formed is directly proportional to the amount of ADA present in the sample.

The procedure was as follows: $i$ ). All the reagent and sample was brought to room temperature before use; ii). Working phenol reagent and working hypochlorite reagents were prepared; iii). Calorimeter was set with filter of $570-630 \mathrm{~nm}$ at $37^{\circ} \mathrm{C}$. $i v$ ). Four clean dry test tube were labeled as reagent blank (B), standard(S), sample blank (SB), sample or test $(\mathrm{T}) ; v)$. Addition of reagent into these test tube was done as follows; $v i$ ). It was mixed well and incubated at $37^{\circ} \mathrm{C}$ for exactly 60 minutes, and then following reagents were added; vii). It was mixed well and incubated at $37^{\circ} \mathrm{C}$ for 30 minutes; viii). Absorbance (optical densities) of the reagent blank (Abs.B), standard (Abs.S), sample blank (Abs.SB), sample or test (Abs.T) was measured by using the calorimeter; $i x$ ).

\section{Calculation}

All collected data was entered into a SPSS 17.0 Analysis was done using statistical tests such as independent Sample t-test, one Sample t-test and one way ANOVA followed by Tuckey's POST HOC test wherever applicable. The level of significance was set at 5\%. All $\mathrm{p}$-values less than 0.05 were treated as a significant.

\section{Results}

Table1 provides a comparison of mean serum ADA level of control group with OSCC group and Table 2 multiple comparisons of mean serum ADA level of control group with different histopathological grades of OSCC.

Photmicrographs of histopathology of different lesions are given in Figure 1. When serum ADA level of control group was compared with well moderate and poorly differentiated OSCC group $\mathrm{P}$ value was $0.00(<0.05)$ hence was statistically highly significant. When serum ADA level of well differentiated group was compared with moderately and poorly differentiated OSCC group P value was $0.00(<0.05)$ and hence was statistically highly significant. When serum ADA level of moderately differentiated group was compared with poorly differentiated OSCC group P value was $0.164(>0.00)$ and hence was statistically insignificant.

Figure 2 demonstrates mean serum ADA levels in each group. There is an increase in serum ADA levels from control to poorly differentiated OSCC.

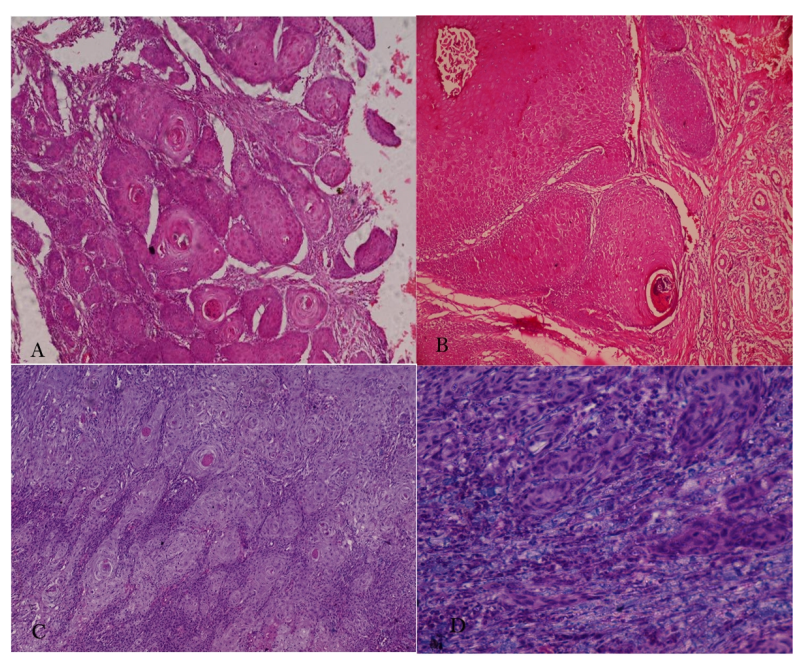

Figure 1. Histopathological Examination of OSCCs. A \& B: Well differentiated, C: Moderately differentiated and D: Poorly differentiated grade

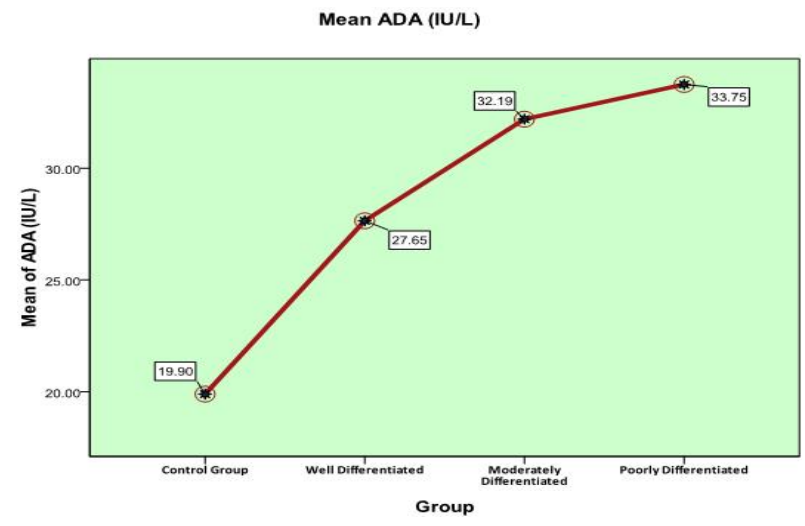

Figure 1. Mean serum ADA Levels in Each Group. There is an increase in serum ADA levels from control to poorly differentiated OSCC

\section{Table 1. Comparison of Mean Serum ADA Level of Control Group with OSCC Group}

\begin{tabular}{ccccccc}
\hline Group & $\mathrm{n}$ & Mean & SD & Mean Difference & t-stat & P-Value \\
\hline OSCC Group & 90 & 31.1953 & 4.171 & 11.297 & 14.34 \\
Control Group & 90 & 19.8983 & 1.106 & & $<0.001 * *$ \\
\hline
\end{tabular}

** Highly significant difference 
Table 2. Multiple Comparisons of Mean Serum ADA Level of control group with different histopathological grades of OSCC

\begin{tabular}{|c|c|c|c|c|}
\hline \multirow{2}{*}{ (I) Group } & \multirow{2}{*}{ (J) Group } & \multirow{2}{*}{ Mean Difference (I-J) } & \multirow{2}{*}{ Std. Error } & Sig. \\
\hline & & & & (p value) \\
\hline \multirow{3}{*}{ Control Group } & Well Differentiated & $-7.75067 *$ & 0.89873 & OPROVIDE! \\
\hline & Moderately Differentiated & $-12.29367 *$ & 0.89873 & 0 \\
\hline & Poorly Differentiated & $-13.84667 *$ & 0.89873 & 0 \\
\hline \multirow{3}{*}{ Well Differentiated } & Control Group & $7.75067 *$ & 0.89873 & 0 \\
\hline & Moderately Differentiated & $-4.54300^{*}$ & 1.10072 & 0 \\
\hline & Poorly Differentiated & $-6.09600 *$ & 1.10072 & 0 \\
\hline \multirow{3}{*}{ Moderately Differentiated } & Control Group & $12.29367 *$ & 0.89873 & 0 \\
\hline & Well Differentiated & $4.54300 *$ & 1.10072 & 0 \\
\hline & Poorly Differentiated & -1.553 & 1.10072 & 0.164 \\
\hline \multirow{3}{*}{ Poorly Differentiated } & Control Group & $13.84667 *$ & 0.89873 & 0 \\
\hline & Well Differentiated & $6.09600 *$ & 1.10072 & 0 \\
\hline & Moderately Differentiated & 1.553 & 1.10072 & 0.164 \\
\hline
\end{tabular}

\section{Discussion}

OSCC is one of the most common malignancies in the world. Oral and pharyngeal cancer, grouped together is the sixth leading cancer in the world and ranks in the top three in high incidence areas (Saman Warnakulasuriya, 2009). It traditionally caries a poor prognosis with more than $50 \%$ of the cases diagnosed at an advanced stage, with the five year survival rate remaining low at $~ 30$ to $50 \%$ (Saman Warnakulasuriya, 2009). Lack of noninvasive and reliable screening test has been quoted as the main reason. In this scenario minimally invasive cancer specific tests are urgently sought and recently serological tumor markers have been included and actively pursued to obtain an easy, simple and reliable diagnostic tool for the detection of oral cancer. Serum analysis of various biochemical markers in cancer has diagnostic and prognostic significance. There are a number of tumor markers studied which can be used in the diagnosis and prognosis of oral cancer, however none of these markers have a consistent sensitivity and specificity and hence the pursuit for newer markers has continued.

It has been known for many years that the enzyme complement of a tumor cell differs in many ways from that of its normal counterpart reflecting its altered metabolism. Elevated enzyme levels in cancer patients denote aggressiveness of cancer, unchanged or increasing levels indicating a lack of response, also decrease to normal levels following successful treatment (M.M. Suchitra et al., 2009). Serum enzymes are useful for monitoring the effects of therapy, to detect recurrences and also have prognostic value as their level frequently reflects tumor burden. ADA an enzyme of the purine salvage pathway is widely distributed in tissues. Many studies have demonstrated alterations of ADA activity in the tumor tissue and serum in patients with lung, head and neck, breast and ovarian cancer (Mini, 1995; Selda et al, 1996; Pragathi et al., 2005; Ashok et al., 2008). It catalyses hydrolytic deamination of adenosine to inosine and 2"deoxy adenosine to 2"deoxy inosine respectively. Numerous studies have documented an increase of ADA in very rapidly growing malignancies, where it has been documented as a serological tumor marker, while slow growing well differentiated tumors do not express pronounced ADA activity (Balis, 1985).

The normal serum ADA level in healthy individuals ranges from 18 to $22 \mathrm{IU} / \mathrm{L}$. In our study we found that the serum ADA level in all control group individuals were within the normal range (mean 19.90 IU/L). Mean serum ADA level in study group was $31.20 \mathrm{IU} / \mathrm{L}$. Our results showed the statistically significant increased serum ADA levels in study group as compared to control group which is in agreement with the study done by Harbans Lal (1987), R. Mishra (2000), Kalcioglu MT (2004), Ashok K.J (2008) and Rakesh Dhankhar (2011). Kalcioglu MT in 2004 showed that serum ADA level was increased significantly in OSCC, thus suggested that serum ADA activity may be helpful in the diagnosis and follow-up of head and neck cancers. Rakesh Dhankhar (2011) found that levels of adenosine deaminase, uric acid, and C-reactive protein were significantly higher in patients of head and neck cancers as compared to the levels in controls.

Various studies on gastric, colorectal, breast, bladder, and ovarian cancers reported the increase in serum ADA level. Mini Walia et al. (1995) found a significantly $(p<0.001)$ increased level of serum ADA in breast cancer patients compared to normal individuals, thus they suggested that serum ADA can be a useful parameter for diagnosing breast cancer and for monitoring its progression. Pragathi, P. et al in 2005 showed a significant increase in serum ADA levels in the ovarian cancer group but not in the benign group when compared to controls. They suggested that the elevated levels were certainly due to the presence of cancer cells, and thus it can serve as a parameter to differentiate malignant conditions from the benign tumors of the ovary. 
In our study mean serum ADA level in well, moderate and poorly differentiated grade were $27.3 \mathrm{IU} / \mathrm{L}, 31.8$ $\mathrm{IU} / \mathrm{L}$ and $33.5 \mathrm{IU} / \mathrm{L}$ respectively. We found that serum ADA level gradually increased from well, moderate to poorly differentiated OSCC grade. Statistically significant difference was present between well \& moderate grade, and between well \& poor grade, but insignificant difference between moderate $\&$ poor grade. In the literature previous studies mentioned the correlation of serum ADA level with different clinical stages of oral cancer. To the best of our knowledge no study correlated the serum ADA levels with different histopathological grades. Harbans Lal et al in 1987 evaluated the serum ADA in 40 OSCC patients of different stages and found the ADA activity is increased with advancement in the clinical stage of the cancer. Study done by R. Mishra et al. (2000) found significant correlation between increased levels of ADA with lymph node involvement and concluded that this may help in assessing the decrease in tumor mass and improvement in patient \& clinical condition. Ashok et al in 2008 found that there was a highly significant correlation between the serum ADA level and the increasing disease stage (severity of the disease), the tumor status and metastasis of the tumor to the neck nodes. They concluded that Serum ADA levels can be used as one of the diagnostic tools in head and neck cancer.

ADA is an enzyme of the purine salvage pathway. ADA is present on the cell surface as well as intra-cellularly, but it does not have its own transmembrane domain and is associated with CD26, a surface glycoprotein with dipeptidyl peptidase IV activity (Franco, 1997). Due to the rapid growth, solid tumors routinely experience severe hypoxia and necrosis leading to adenine nucleotide degradation and adenosine release (Linden, 2006). The released adenosine constitutes supportive environment for tumor growth by means of protection against ischemia. At the same time it stimulates the growth and angiogenesis as well as suppresses immune response. So increase in adenosine production leads to increase in production of ADA. Also, in cancer there is an increased turnover of malignant cells and an associated increase in nucleotide metabolism leading to an increase in purine metabolizing enzymes. ADA is particularly sensitive to stimulation by growth factors and cytokines during rapid tissue proliferation such as IL-2, IL12 and INF $\gamma$ which increases during malignancy (Ashok et al., 2008).

Results of our study showed that serum ADA level increases significantly in OSCC as compared to control group. It shows that nucleotide (purine \& pyrimidine) metabolism increases in OSCC due to increase in DNA turnover, and serum level increases because of leakage of enzyme from primary malignant cells and lymphatic metastasis. In the literature it is mentioned that the prognosis of OSCC worsened from well to poorly differentiated grade, also serum ADA is known to increase with disease progression. In our study, serum ADA level increased from well to moderate to poorly differentiated OSCC. Therefore it can be suggested that serum ADA can be used as a diagnostic and also prognostic biomarker for OSCC. Also the simplicity of measuring ADA activity combined with its cost effectiveness gives an added advantage to consider ADA as a tumor marker in oral cancer. However, during evaluation of serum ADA level one has to keep in mind that the level of these markers altered in different systemic conditions such as various cancers, infections etc, and so these factors should be ruled out.

Thus, from our study observations it is seen that, serum ADA level increases in OSCC, also the level of these markers increases according to histopathological grade. Hence we propose that serum ADA can be used as a diagnostic as well as prognostic bio-marker in OSCC.

\section{References}

Ashok KJ, Pinto GJO, Kavitha AK, et al (2008). The diagnostic and prognostic value of serum adenosine deaminase levels in head and neck cancer. J Clin Diag Res, 3, 833-37.

Balis EM (1985). Adenosine deaminase and malignant cells. Ann NY Acad sci, 451, 142-9.

Franco R (1997). Cell surface adenosine deaminase: much more than an ectoenzyme. Prog Neurobiol, 52, 283-94.

Ferlay J, Pisani P, Parkin DM (2004). Globocan. Cancer incidence, mortality and prevalence worldwide. Lyon: IARC Press Guesti G (1974). Adenosine deaminase In: Bergmeyer $\mathrm{HU}$ editor. Methods of enzymatic analysis, $2^{\text {nd }} \mathrm{Ed}, 2$, New York: Academic press Inc, 1092-9.

Harbans lal, SK Munjal, Umesh Wig, et al (1987). Serum enzymes in head and neck cancer III. J Laryngol Otol, 101, 1062-5.

Kalcioglu MT (2004). Adenosine deaminase, xanthine oxidase, superoxide dismutase, glutathione peroxidase activities and malondialdehyde levels in the serum of patients with head and neck carcinoma. Kulak Burun Bogaz Ihtis Derg, 12, 16-22.

Kerawala CJ (2010). Complications of head and neck cancer surgery: Prevention and management. Oral Oncol, 46, 433-5.

Linden J (2006). Adenosine metabolism and cancer. Am J Physiol Cell Physiol, 17, 1-7.

Mini walia, Mridula Mahajan, K Singh (1995). serum adenosine deaminase, 5' - nucleotidase \& alkaline phosphatase in Breast cancer Patients. Indian J Med Res, 101, 247-9.

Suchitra MM, Prabhakar Reddy E, Muni Sudhakar G, et al (2009). Evaluation of serum adenosine deaminase as a tumor marker in gastric cancer. Res J Medicine Med Sci, 4, 411-4.

Pragathi P, Bharath Kumar,Amar Kumar P, et al (2005). Evaluation of serum adenosine deaminase and 5 '-nucleotidase activities as probable markers in ovarian cancer. Indian J Clin Bio, 20, $195-7$.

Mishra R, Agarwal MK, Chansuria JPN (2000). Serum adenosine deaminase levels as an index of tumor growth in head and neck malignancy. Indian J Otolaryngol Head Neck Surg, 52, 360-3.

Rakesh Dhankhar, Kiran Dahiya, Tarun Kumar Sharma, et al (2011). Diagnostic significance of adenosine deaminase, uric acid and C-reactive protein levels in patients of head and neck carcinoma. Clin Lab, 57, 795-8.

Dermirtas S (1996). Adenosine deaminase, 5'-nucleotidase, guanase and cytidine deaminase activites in gastric and breast-cancer. SDV Tip Fakultesi Dergisi, 3, 1-4.

St. John, MAR, Li Y, Zhou X, et al (2004). Interleukin 6 and interleukin 8 as potential biomarkers for oral cavity and oropharyngeal squamous cell carcinoma. Arch Otolaryngol Head Neck Surg, 103, 929-35.

Warnakulasuriya S (2009). Global epidemiology of oral and pharyngeal cancer. Oral Oncol, 45, 309-16. 